\title{
ORDERED PROBABILISTIC METRIC SPACES
}

\author{
D. C. KENT AND G. D. RICHARDSON
}

(Received 1 August 1986; revised 5 March 1987)

Communicated by J. H. Rubinstein

\begin{abstract}
Probabilistic quasi-metric spaces are introduced and used to define ordered probabilistic metric spaces. The latter spaces arise naturally in the study of probability and statistics; they closely resemble the uniform ordered spaces of $\mathrm{L}$. Nachbin. A procedure is described for constructing ordered probabilistic metric spaces from quasi-simple spaces, and a completion theory is developed simultaneously for probabilistic quasi-metric spaces and ordered probabilistic metric spaces.

1980 Mathematics subject classification (Amer. Math. Soc.) (1985 Revision): 54 A 05, 54 A 20, 60 A 05.

Keywords and phrases: ordered probabilistic metric space, uniform space, Cauchy filter, completion.
\end{abstract}

\section{Introduction}

A probabilistic metric space is a generalized metric space appropriate to the study of situations in which "distances" are measured in terms of distribution functions rather than non-negative real numbers. In many cases, a probabilistic metric space is endowed with an intrinsic order and questions naturally arise concerning the interaction between the order and the generalized metric, possible ways to extend the order to a completion space, etc..

An effective way to define compatibility between a uniform structure and an order structure on the same set $S$ was introduced by L. Nachbin [2]. A quasiuniformity (which Nachbin calls a "semi-uniform structure") is a filter $\mathscr{U}$ on

(c) 1989 Australian Mathematical Society $0263-6115 / 89 \$ A 2.00+0.00$ 
$S \times S$ which is required to satisfy all the properties of uniformity except the symmetry property $\mathscr{U}=\mathscr{U}^{-1}$. Nachbin defines a triple $(S, \mathscr{V}, \leq)$, where $\mathscr{V}$ is a uniformity and $\leq$ a partial order on $S$, to be a uniform ordered space if there is a quasi-uniformity $\mathscr{U}$ on $S$, such that $\mathscr{V}=\mathscr{U} \wedge \mathscr{U}^{-1}$ is the smallest uniformity containing $\mathscr{U}$, and $p \leq q$ if and only if $(p, q) \in U$ for all $U \in \mathscr{U}$. In a similar way, a (probabilistic) quasi-metric on $S$ gives rise to an ordered (probabilistic) metric space.

Probabilistic quasi-metric spaces, ordered probabilistic metric spaces, and the relationships between them are the subject of Sections 2 and 3. Section 4 deals with quasi-simple spaces; these are probabilistic quasi-metric spaces derived from ordinary quasi-metrics. It is shown in Section 4 that every ordered probabilistic metric space is compatible with a quasi-simple space. It is also shown in this section that a probabilistic metric space with a partial order is an ordered probabilistic metric space if and only if the associated strong uniformity and the given order constitute a uniform ordered space. In Section 5, a completion theory, similar to that of Sherwood [4] for probabilistic metric spaces, is developed for probabilistic quasi-metric spaces and ordered probabilistic metric spaces.

\section{Probabilistic quasi-metric spaces}

A distance distribution function $F$ is a non-decreasing function from $R^{+}=$ $[0, \infty]$ into $[0,1]$, which is left continuous on $(0, \infty)$ and takes on the values $F(0)=0$ and $F(\infty)=1$. The set of all distance distribution functions, denoted by $\Delta^{+}$, is equipped with the modified Lévy metric $d_{L}$ (see page 45 of [4]). Convergence in the metric space $\left(\Delta^{+}, d_{L}\right)$ is characterized by: $\left(F_{n}\right) \rightarrow F$ if and only if $\left(F_{n}(x)\right) \rightarrow F(x)$ in $R$, whenever $x$ is a point of continuity of $F$. The metric space $\left(\Delta^{+}, d_{L}\right)$ is compact, and $\Delta^{+}$is partially ordered by the usual (pointwise) order for real-valued functions.

Let $\varepsilon_{0}$ be the element of $\Delta^{+}$defined by

$$
\varepsilon_{0}(x)= \begin{cases}0, & x=0 \\ 1, & x>0\end{cases}
$$

A triangle function $\tau$ is defined to be a binary operation on $\Delta^{+}$which is commutative, associative, non-decreasing in each component, and has $\varepsilon_{0}$ as its identity. All triangle functions considered in this paper will, in addition, be assumed to be continuous with respect to the topology induced by metric $d_{L}$. Given $t>0$, let $N_{\varepsilon_{0}}(t)=\left\{F \in \Delta^{+}: d_{L}\left(F, \varepsilon_{0}\right)<t\right\}$ denote a basic neighborhood of $\varepsilon_{0}$ in this topology. 
DEFINITION 2.1. Let $S$ be a non-empty set, and let $\tau$ be a triangle function on $\Delta^{+}$. A function $\mathscr{F}: S \times S \rightarrow \Delta^{+}$is a probabilistic quasi-metric if the following conditions are satisfied for all $p, q, r$ in $S$ :

$(\mathrm{PM})_{1} \mathscr{F}(p, p)=\varepsilon_{0}$

$(\mathrm{PM})_{2}$ if $\mathscr{F}(p, q)=\varepsilon_{0}$ and $\mathscr{F}(q, p)=\varepsilon_{0}$, then $p=q$

$(\mathrm{PM})_{3} \mathscr{F}(p, q) \geq \tau(\mathscr{F}(p, r), \mathscr{F}(r, q))$.

If $\mathscr{F}$ is a probabilistic quasi-metric, then the triple $(S, \mathscr{F}, \tau)$ is called a probabilistic quasi-metric space (abbreviated $P Q M$ space). A probabilistic quasi-metric $\mathscr{F}$ is called a probabilistic metric if it satisfes the symmetry condition

$(\mathrm{PM})_{4} \mathscr{F}(p, q)=\mathscr{F}(q, p)$ for all $p, q$ in $S$.

In the latter case, $(S, \mathscr{F}, \tau)$ is a probabilistic metric space (abbreviated $P M$ space).

We shall often adopt the common practice of writing $F_{p q}$ in place of $\mathscr{F}(p, q)$.

In [1], P. S. Marcus gives an example of a PQM space based on stationary Markov chains which is not a PM space.

An excellent treatment of PM spaces is given in the book co-authored by B. Schweizer and A. Sklar [4]. One familiar concept associated with a PM space $(S, \mathscr{F}, \tau)$ (recall that $\tau$ is always assumed to be continuous) is the strong uniformity $\mathscr{U}$ on $S$. For each $t>0$, let $U(t)=\left\{(p, q) \in S \times S: F_{p q} \in N_{\varepsilon_{0}}(t)\right\}$; the sets $\{U(t): t>0\}$ constitute a filter base for $\mathscr{U}$. If $(S, \mathscr{F}, \tau)$ is a PQM space, it is easy to verify that the filter $\mathscr{U}$ constructed in this way is a quasi-uniformity, which we shall call the strong quasi-uniformity determined by $(S, \mathscr{F}, \tau)$. From the strong quasi-uniformity, we obtain the strong topology $\sigma$ associated with $\mathscr{F}$ which has for its neighborhood base at $p \in S$ the collection $\left\{U_{p}(t): U \in \mathscr{U}\right.$, $t>0\}$, where $U_{p}(t)=\{q \in S:(p, q) \in U(t)\}$.

We can also associate with any PQM space $(S, \mathscr{F}, \tau)$ an "inverse PQM space" $\left(S, \mathscr{F}^{\prime}, \tau\right)$, where $\mathscr{F}^{\prime}(p, q)=\mathscr{F}(q, p)$ for all $p, q$ in $S$. If $\mathscr{U}$ is the strong quasiuniformity for $\mathscr{F}$, then $\mathscr{U}^{-1}$ is the strong quasi-uniformity for $\mathscr{F}$. Also associated with $\mathscr{F}$ is the uniformity $\hat{\mathscr{U}}=\mathscr{U} \vee \mathscr{U}^{-1}$. From $\mathscr{U}, \mathscr{U}^{-1}$, and $\hat{\mathscr{U}}$ we derive in the natural way the topologies $\sigma, \sigma^{1}, \hat{\sigma}$. In the next section, we define a probabilistic metric $\hat{\mathscr{F}}$ associated with $\mathscr{F}$, such that $\hat{\mathscr{U}}$ (respectively, $\hat{\sigma}$ ) is the strong uniformity (respectively, strong topology) for $\hat{F}$. Some properties of the topologies $\sigma, \sigma^{1}$, and $\hat{\sigma}$ are given in the next two propositions; the straightforward proofs are omitted. The symbols $\wedge$ and $\vee$ are used throughout this work to denote the greatest lower bound and least upper bound, respectively.

PROPOSITION 2.1. Let $(S, \mathscr{F}, \tau)$ be a PQM space, let $\left(p_{n}\right)$ be a sequence in $S$, and $p \in S$.

(1) $\left(p_{n}\right) \rightarrow p$ in $(S, \sigma)$ if and only if $F_{p p_{n}} \rightarrow \varepsilon_{0}$ in $\left(\Delta^{+}, d_{L}\right)$.

(2) $\left(p_{n}\right) \rightarrow p$ in $\left(S, \sigma^{\prime}\right)$ if and only if $F_{p_{n} p} \rightarrow \varepsilon_{0}$ in $\left(\Delta^{+}, d_{L}\right)$. 
(3) $\left(p_{n}\right) \rightarrow p$ in $(S, \hat{\sigma})$ if and only if $F_{p p_{n}} \wedge F_{p_{n} p} \rightarrow \varepsilon_{0}$ in $\left(\Delta^{+}, d_{L}\right)$ or, equivalently if and only if (1) and (2) hold.

Given a non-empty subset $A$ of $S$, let $F_{p A}=\sup \left\{F_{p q}: q \in A\right\}$ and $F_{A p}=$ $\sup \left\{F_{q p}: q \in A\right\} ;$ note that $F_{p A}$ and $F_{A p}$ are both elements of $\Delta^{+}$.

Proposition 2.2. Let $(S, \mathscr{F}, \tau)$ be a PQM space. A non-empty subset $A$ of $S$ is $\sigma$-closed (respectively, $\sigma^{\prime}$-closed) if and only if $F_{p A} \neq \varepsilon_{0}$ (respectively, $\left.F_{A p} \neq \varepsilon_{0}\right)$ whenever $p \notin A$.

In addition to generating certain quasi-uniformities and topologies on $S$, a probabilistic quasi-metric $\mathscr{F}$ also determines an order on $S$ according to the rule: $p \leq q$ if and only if $F_{p q}=\varepsilon_{0}$. This is also the order induced by the strong quasi-uniformity associated with $\mathscr{F}$. If $\mathscr{F}$ is a probabilistic metric, the induced order is the discrete (or trivial) order: $p \leq q$ if and only if $p=q$.

\section{Ordered probabilistic metric spaces}

DEFINITION 3.1. A quadruple $(S, \mathscr{G}, \tau, \leq)$ is an ordered probabilistic metric space (abbreviated $O P M$ space) if $(S, \mathscr{G}, \tau)$ is a PM space, and there is a PQM space $\left(S, \mathscr{F}, \tau^{\prime}\right)$ such that the following conditions are satisfied:

(i) If $\mathscr{U}$ is the strong quasi-uniformity for $\mathscr{F}$, then $\hat{\mathscr{U}}=\mathscr{U} \vee \mathscr{U}^{-1}$ is the strong uniformity for $\mathscr{G}$.

(ii) $(S, \leq)$ is a poset with partial order determined by $\mathscr{F}$.

A PQM space $\left(S, \mathscr{F}, \tau^{\prime}\right)$ and an OPM space $(S, \mathscr{G}, \tau, \leq)$ which are related in the manner specified in Definition 3.1 are said to be compatible. Any PM space $(S, \mathscr{G}, \tau)$ with the discrete order can be regarded as an OPM space, where a compatible PQM space is $(S, \mathscr{F}, \tau)=(S, \mathscr{G}, \tau)$. If $(S, \mathscr{G}, \tau, \leq)$ is an OPM space, then $(S, \hat{\mathscr{U}}, \leq)$ is a uniform ordered space. Two OPM spaces are uniformly equivalent if they induce the same uniform ordered space; uniformly equivalent OPM spaces must have the same underlying set, but their triangle functions may be different.

It is obvious from Definition 3.1, that every OPM space is compatible with a $\mathrm{PQM}$ space. We next describe a procedure for constructing an OPM space from a given $\mathrm{PQM}$ space. Starting with a PQM space $(S, \mathscr{F}, \tau)$, recall that $\mathscr{F}^{\prime}$ is the probabilistic quasi-metric inverse to $\mathscr{F}$.

Let $\tau^{*}$ denote any (continuous) triangle function which dominates $\tau$ (see [4], page 209), and define $\mathscr{F}^{*}(p, q)=\tau^{*}\left(\mathscr{F}(p, q), \mathscr{F}^{\prime}(p, q)\right)$. The triangle function defined by $\hat{\tau}(F, G)=F \wedge G$ dominates $\tau$ and note that $\mathscr{F} *$ reduces to $\mathscr{F} \wedge \mathscr{F}$ in this case. Define $\hat{\mathscr{F}}=\mathscr{F} \wedge \mathscr{F}^{\prime}$. Moreover, $\tau \gg \tau([4]$, page 210). 
PROPOSITION 3.2. If $(S, \mathscr{F}, \tau)$ is a $P Q M$ space and $\leq$ the partial order determined by $\mathscr{F}$, then $\left(S, \mathscr{F}^{*}, \tau, \leq\right)$ is an OPM space compatible with $(S, \mathscr{F}, \tau)$. In particular, $(S, \hat{F}, \tau, \leq)$ and $(S, \mathscr{F}, \tau)$ are compatible.

PROOF. It must be shown that $\left(S, \mathscr{F}^{*}, \tau\right)$ is a PM space and $\mathscr{V}=\mathscr{U} \vee \mathscr{U}^{-1}$, where $\mathscr{V}$ is the strong uniformity for $\mathscr{F}^{*}$ and $\mathscr{U}$ is the strong quasi-uniformity for $\mathscr{F}$.

First, note that the symmetry of $\mathscr{F}^{*}$ is obvious and that $F_{p p}^{*}=\tau^{*}\left(F_{p p}, F_{p p}\right)=$ $\varepsilon_{0}$. Next, if $F_{p q}^{*}=\varepsilon_{0}$, then $F_{p q} \wedge F_{q p} \geq \tau^{*}\left(F_{p q}, F_{q p}\right)=\varepsilon_{0}$, which implies that $F_{p q}=F_{q p}=\varepsilon_{0}$ and thus $p=q$. Let us use the definition of $\tau^{*} \gg \tau$ to prove the triangle inequality. We have $\tau\left(F_{p r}^{*}, F_{r q}^{*}\right)=\tau\left(\tau^{*}\left(F_{p r}, F_{r p}\right), \tau^{*}\left(F_{r q}, F_{q r}\right)\right) \leq$ $\tau^{*}\left(\tau\left(F_{p r}, F_{r q}\right), \tau\left(F_{r p}, F_{q r}\right)\right) \leq \tau^{*}\left(F_{p q}, F_{q p}\right)=F_{p q}^{*}$. Hence, $\left(S, F^{*}, \tau\right)$ is a PM space.

Note $\hat{\tau} \gg \tau^{*} \gg \tau$. Using the continuity of $\tau$ at $\left(\varepsilon_{0}, \varepsilon_{0}\right)$, it is straightforward to verify that the strong uniformities for $\tau^{*}=\tau$ and $\tau^{*}=\hat{\tau}$ coincide. Moreover, for $\tau^{*}=\hat{\tau}, \mathscr{V}=\mathscr{U} \vee \mathscr{U}^{-1}$ and thus $(S, \mathscr{F}, \tau)$ and $\left(S, \mathscr{F}^{*}, \tau, \leq\right)$ are compatible whenever $\tau^{*} \gg \tau$.

EXAMPLES 3.3. To illustrate the above, we show that the set of all equivalence classes of random variables defined on a given probability space and equipped with partial order less than or equal almost surely is an OPM space. The probabilistic metric on this space induces convergence in probability.

Let $(\Omega, A, P)$ denote a probability space and denote by $[X]$ the set of all random variables defined on $(\Omega, A, P)$ which are equal to $X$ almost surely. Suppose that $S$ is the set of all such equivalence classes.

For each $p=[X], q=[Y]$, define $F_{p q}$ to be the distribution function of the random variable $Z=(X-Y) .1_{\{X \geq Y\}}$, where

$$
\left\{\begin{array}{cl}
1(w) \\
\{X \geq Y\}
\end{array}= \begin{cases}1, & \text { if } X(w) \geq Y(w) \\
0, & \text { otherwise }\end{cases}\right.
$$

$w \in \Omega$. Since $Z$ is a non-negative random variable, then $F_{p q} \varepsilon \Delta^{+}$. Clearly, $F_{p p}=\varepsilon_{0}$. Next, suppose that $F_{p q}=F_{q p}=\varepsilon_{0}$. Then $Z=0$ almost surely and thus $X \leq Y$ almost surely. Similarly, $Y \leq X$ almost surely, and hence $p=q$.

Let $I=[0,1]$ and define $W: I \times I \rightarrow I$ by $W(a, b)=(a+b-1) \vee 0$ and let $\tau_{W}(F, G)(x)=\sup \{W(F(y), F(z)) \mid y, z \in I, y+z=x\}$, where $F, G \in \Delta^{+}$and $x \geq 0$. The proof that $F_{p q} \geq \tau_{W}\left(F_{p r}, F_{r q}\right)$ is given in Theorem 9.1.2 of [4] and thus $\left(S, F, \tau_{W}\right)$ is a PQM space. Recall that $p=[X] \leq q=[Y]$ if and only if $F_{p q}=\varepsilon_{0}$. Again, this happens if and only if $X \leq Y$ almost surely, and thus, this is the partial order induced by the PQM space $\left(S, \mathscr{F}, \tau_{W}\right)$.

Let us characterize convergence in $(S, \hat{\sigma})$. Denote by $p_{n}=\left[X_{n}\right], p=[X]$, and $Z_{n}=\left(X_{n}-X\right) \cdot 1_{\left\{X_{n} \geq X\right\}}$. Then, for $t>0, F_{p_{n}}(t)=P\left\{Z_{n}<t\right\} \rightarrow 1$ if and only 
if $P\left\{X_{n}-X<t\right\} \rightarrow 1$. Similarly, $F_{p p_{n}}(t) \rightarrow 1$ if and only if $P\left\{X-X_{n}<t\right\} \rightarrow 1$, and thus, $p_{n} \rightarrow p$ in $(S, \hat{\sigma})$ if and only if $X_{n} \rightarrow X$ in probability.

Let us conclude this section with another probabilistic quasi-metric which induces the same order on $S$ above. Define, for each $p=[X], q=[Y], G_{p q}$ to be the distribution function of the random variable $1_{\{X>Y\}}$. It can by shown that $\left(S, \mathscr{G}, \tau_{W}\right)$ is a PQM space. Moreover, note that $p \leq q$ if and only if $P\left\{1_{\{X>Y\}}<t\right\}=1$ for each $t>0$. Hence $p \leq q$ if and only if $X \leq Y$ almost surely, which agrees with the order induced in the example above. However, if $p_{n}=\left[X_{n}\right], p=[X]$, then $p_{n} \rightarrow p$ in $(S, \hat{\sigma})$ if and only if $P\left\{1_{\left\{X_{n}>X\right\}}<t\right\} \rightarrow 1$ and $P\left\{1_{\left\{X>X_{n}\right\}}<t\right\} \rightarrow 1$, for each $t>0$. This implies that $p_{n} \rightarrow p$ in $(S, \hat{\sigma})$ if and only if $P\left\{X_{n}=X\right\} \rightarrow 1$. The topology here is strictly finer than that of the example above even though the partial orders agree.

\section{Quasi-simple spaces}

A quasi-simple space is a PQM space derived (as in [4, Section 8.4] from a quasi-metric $\delta$ on $S$. It turns out that in the definition of OPM space (Definition $3.1)$, there is no loss of generality if the PQM space $\left(S, \mathscr{F}, \tau^{\prime}\right)$ is assumed to be a quasi-simple space.

Let $\tau_{\mathscr{A}}$ be the triangle function defined as follows: $\tau_{\mathscr{K}}(F, G)(x)=\sup \{F(y) \wedge$ $G(z): y \geq 0, z \geq 0, y+z=x\}$. A quasi-metric $\delta$ on $S$ is a function from $S \times S$ into $[0, \infty)$, which is required to satisfy the following proper;ties: (1) $\delta(p, p)=0$ for each $p \in S$; (2) if $\delta(p, q)=\delta(q, p)=0$, then $p=q$; and (3) $\delta(p, q) \leq \delta(p, r)+\delta(r, q)$ for each $p, q, r$ in $S$. Given a quasi-metric space $(S, \delta)$ and an element $G$ of $\Delta^{+}$which is distinct from $\varepsilon_{0}$; define $\mathscr{F}_{\delta}(p, q)(x)=G(x / \delta(p, q))$ for each $p, q, \varepsilon S$ and $x \geq 0$. A PQM space $(S, \mathscr{F}, \tau)$ is said to be a quasi-simple space if $\tau=\tau_{M}$ and there is a quasi-metric $\delta$ on $S$ such that $\mathscr{F}=\mathscr{F}$. It is shown in $\left[4\right.$, Theorem 8.4.2] that $\mathscr{F}_{\delta}$ is a probabilistic metric relative to the triangle function $\tau_{M}$ whenever $\delta$ is a metric on $S$. This theorem generalizes without difficulty to quasi-metrics. It should be noted that $\mathscr{F}_{\delta}$ depends on $G$ as well as $\delta$.

Proposition 4.1. Let $(S, \delta)$ be a quasi-metric space and let $G$ be an element of $\Delta^{+}$which is distinct from $\varepsilon_{0}$. Then $\left(S, \mathscr{F}_{\delta}, \tau_{M}\right)$ is a $P Q M$ space.

With each quasi-metric $\delta$ on $S$, we associate the metric $\hat{\delta}$ defined by $\hat{\delta}(p, q)=$ $\delta(p, q) \vee \delta(q, p)$ for all $p, q$ in $S$; we also associate the quasi-uniformity $\mathscr{V} \delta$ generated by sets of the form $V(t)=\{(p, q): \delta(p, q)<t\}$ for all $t>0$, and the uniformity $\hat{\mathscr{V}}_{\delta}=\mathscr{V}_{\delta} \vee \mathscr{V}_{\delta}^{-1}$ determined by $\hat{\delta}$. For a given $G \in \Delta^{+}$, the simple spaces generated by $\delta$ and $\hat{\delta}$ are related in the expected way. 
Proposition 4.2. Let $\delta$ be a quasi-metric on $S$ and let $G \in \Delta^{+}$be such that $G \neq \varepsilon_{0}$; let $\mathscr{F}(p, q)(x)=G(x / \delta(p, q))$ for all $x \in[0, \infty]$. Then $\left(S, \hat{F}, \tau_{\mathscr{R}}\right)$ is the simple space determined by $G$ and $\hat{\delta} ;$ in other words, $\hat{\mathscr{F}}(p, q)(x)$ $=G(x / \hat{\delta}(p, q))$, for all $x \in[0, \infty]$.

Proof. Note that $\hat{F}_{p q}(x)=F_{p q}(x) \wedge F_{q p}(x)=G(x / \delta(p, q)) \wedge G(x / \delta(q, p))=$ $G(x / \hat{\delta}(p, q))$, for all $x \in[0, \infty]$.

It is also true that the quasi-uniformity determined by a quasi-metric coincides with the strong quasi-uniformity determined by the quasi-simple space arising from the same quasi-metric.

Proposition 4.3. Let $\delta, G$, and $\mathscr{F}$ be as specified in Proposition 4.2. Assume that $G$ is an element in $\Delta^{+}$which is distinct from $\varepsilon_{0}$ and such that $\lim _{y \rightarrow \infty} G(y)=1$, and let $\mathscr{U}$ be the strong quasi-uniformity for $\left(S, \mathscr{F}, \tau_{\mathscr{M}}\right)$. Then $\mathscr{U}=\mathscr{V}_{\delta}$.

Proof. Let $U(t)=\left\{(p, q): F_{p q} \in N_{\varepsilon_{0}}(t)\right\}$ be a basic set for $\mathscr{U}$, and let $s>0$ be chosen so that $G(t / s)>1-t$; this choice for $s$ is possible because $\lim _{y \rightarrow \infty} G(y)=1$. Then if $(p, q) \in V(s)=\{(p, q): \delta(p, q)<s\}$, it follows that $F_{p q}(t)=G(t / \delta(p, q)) \geq G(t / s)>1-t$, and so $(p, q) \in U(t)$. Thus, $\mathscr{U} \subseteq \mathscr{V}$.

Conversely, let $s$ be given, where $0<s<1$. Since $G \neq \varepsilon_{0}$, we can assume without loss of generality that $G(0+)<1-s$. Denote $G^{\prime}(1-s)=\sup \{x: G(x)<$ $1-s\}$. Note that $G^{\prime}(1-s)>0$, and let $t=s G^{\prime}(1-s) \wedge s$. We will show that $U(t) \subseteq V(s)$. If $(p, q) \notin V(s)$, then $\delta(p, q) \geq s$, and so $F_{p q}(t)=G(t / \delta(p, q)) \leq$ $G\left(s G^{\prime}(1-s) / \delta(p, q)\right) \leq G\left(G^{\prime}(1-s)\right) \leq 1-s \leq 1-t$. Thus, $(p, q) \notin U(t)$. It follows that $\mathscr{U}=\mathscr{V}$.

THEOREM 4.4. Let $(S, \mathscr{G}, \tau)$ be a $P M$ space with strong uniformity $\mathscr{W}$, and let $\leq$ be a partial order on $S$. Then $(S, \mathscr{G}, \tau, \leq)$ is OPM space if and only if $(S, \mathscr{W}, \leq)$ is a uniform ordered space.

ProOF. If $(S, \mathscr{G}, \tau, \leq)$ is an OPM space compatible with the PQM space $\left(S, \mathscr{F}, \tau^{\prime}\right)$ and $\mathscr{U}$ the strong quasi-uniformity for $\left(S, \mathscr{F}, \tau^{\prime}\right)$, then $(S, \mathscr{W}, \leq)$ is the uniform ordered space determined by $\mathscr{U}$.

Conversely, assume $(S, \mathscr{W}, \leq)$ is a uniform ordered space, and let $\mathscr{U}$ be a quasi-uniformity on $S$, such that $\mathscr{W}=\mathscr{U} \vee \mathscr{U}^{-1}$ and $p \leq q$ if and only if $(p, q) \in U$, for all $U \in \mathscr{U}$. Since $\mathscr{W}$ has a countable base, we may assume that $\mathscr{U}$ has a countable base. It follows from [2, Theorem 8] that there is a quasi-metric $\delta$ on $S$, such that $\mathscr{U}=\mathscr{V}_{\delta}$ and $p \leq q$ if and only if $\delta(p, q)=0$.

Let $G$ be any element of $\Delta^{+}$, such that $G \notin \varepsilon_{0}$ and $\lim _{y \rightarrow \infty} G(y)=1$, and define $\mathscr{F}=\mathscr{F}$ as in Proposition 4.1; then $\left(S, \mathscr{F}, \tau_{\mathscr{K}}\right)$ is a quasi-simple space. 
By Proposition 4.3, the strong quasi-uniformity for $\left(S, \mathscr{F}, \tau_{\mathscr{K}}\right)$ is $\mathscr{U}=\mathscr{V}_{\delta}$, and so $\left(S, \mathscr{F}, \tau_{\mathscr{K}}\right)$ generates both the order and the strong uniformity for $(S, \mathscr{G}, \tau, \leq)$. In other words, $(S, \mathscr{G}, \tau, \leq)$ is an OPM space.

The next corollary follows immediately from the proof of Theorem 4.4.

COROLLARY 4.5. Every OPM space is compatible with a quasi-simple space.

A distance distribution function, abbreviated $d d f$, that is continuous and strictly increasing on $[0, \infty]$ is said to be a strict $d d f[4$, page 48$]$. Let $(S, \delta)$ be a metric space, $T$ a strict $t$ norm (see [4], pages 65,66) and define $\tau_{T}(F, G)(z)=$ $\sup \{T(F(x), G(y)) \mid x+y=z\}$. It is proved in [5, Theorem 2] that if $\alpha>1$, there exists a strict $d d f G$ such that $\mathscr{F}(p, q)(x)=G\left(x /(\delta(p, q))^{\alpha}\right)$ defines a probabilistic metric with respect to the triangle function $\tau_{T}$. The verification of condition $(\mathrm{PM})_{3}$ did not require symmetry of $\delta$. Hence, if $(S, \delta)$ is only a quasimetric space, it follows that $\mathscr{F}$, defined above, is a PQM space with respect to the triangle function $\tau_{T}$. In this case $\left(S, \mathscr{F}, \tau_{T}\right)$ is called an $\alpha$-quasi-simple space.

It is straightforward to modify the proof of Proposition 4.3 to show that the strong uniformity of an $\alpha$-quasi-simple space coincides with the quasi-uniformity determined by the quasi-metric. This fact, combined with the proof of Theorem 4.4 , gives the following.

COROLlARY 4.6. Every OPM space $\left(S, \mathscr{F}, \tau_{T}, \leq\right)$, where $T$ is a strict $\tau$ norm, is compatible with an $\alpha$-quasi-simple space, for each $\alpha>1$.

A characterization as to when a uniform space with a partial order is in fact a uniform ordered space is given in [2, Theorem 10]. In particular, $(S, \mathscr{W}, \leq)$ is a uniform ordered space when it is a sup-lattice such that the map $(p, q) \rightarrow p \vee q$ is uniformly continuous [2, Proposition 11].

\section{Completions of OPM spaces}

A completion theory for PM spaces has been developed by Sherwood (see [3], [4]). Completing an OPM space involves extending the order of the original space to its Sherwood completion, and this requires completing a compatible PQM space.

DEFINITION 5.1. Let $(S, \mathscr{G}, \tau, \leq)$ be an OPM space. Then $\left(S^{*}, \mathscr{G}^{*}, \tau, \leq^{*}\right)$ is an OPM completion of $(S, \mathscr{G}, \tau, \leq)$ if $\left(S^{*}, \mathscr{G}^{*}, \tau\right)$ is a probabilistic metric completion of $(S, \mathscr{G}, \tau)$ (see Section 12.5 of [4]) and the embedding of $S$ into $S^{*}$ is an order isomorphism. 
We shall show that every PQM space has a PQM completion which induces an OPM completion of any compatible OPM space. Let $g: S_{1} \times S_{2} \rightarrow T$ be any function and let $A_{i}$ be a subset of $S_{i}, i=1,2$. Then $g\left(A_{1} \times A_{2}\right)$ denotes the subset $\left\{g\left(a_{1}, a_{2}\right) \mid a_{i} \in A_{i}\right\}$ and, moreover, if $\Phi_{i}$ is a filter on $S_{i}$, then $g\left(\Phi_{1} \times \Phi_{2}\right)$ denotes the filter $\left\{A \mid g\left(A_{1} \times A_{2}\right) \subseteq A \subseteq T\right.$, for some $\left.A_{i} \in \Phi_{i}\right\}, i=1,2$. Recall that a filter converges to an element in a topological space provided it contains each neighborhood of this element.

Let $(S, \mathscr{F}, \tau)$ be a PQM space. A filter $\Phi$ on $S$ is defined to be a $\mathscr{F}$-Cauchy filter if and only if $\mathscr{F}(\Phi \times \Phi) \rightarrow \varepsilon_{0}$ in $\left(\Delta^{+}, d_{L}\right)$. Equivalently, $\Phi$ is a $\mathscr{F}$-Cauchy filter if and only if $\mathscr{U} \subseteq \Phi \times \Phi$, where $\mathscr{U}$ is the strong quasi-uniformity for $\mathscr{F}$. Note that the statements $\mathscr{U} \subseteq \Phi \times \Phi, \mathscr{U}^{-1} \subseteq \Phi \times \Phi$, and $\hat{\mathscr{U}} \subseteq \Phi \times \Phi$ are all equivalent. Thus there is no difference between $\mathscr{F}$-Cauchy filters, $\mathscr{F}^{\prime}$-Cauchy

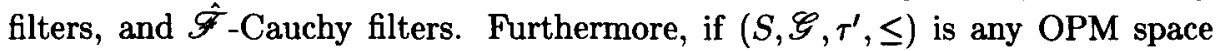
with which $(S, \mathscr{F}, \tau)$ is compatible, then the $\mathscr{F}$-Cauchy filters are the same as the $\mathscr{G}$-Cauchy filters since $\hat{\mathscr{U}}$ is the strong uniformity for $\mathscr{G}$. For simplicity, we shall henceforth refer to the "F -Cauchy filters" simply as "Cauchy filters."

Two Cauchy filters $\Phi$ and $\Psi$ are equivalent if and only if $\Phi \cap \Psi$ is also a Cauchy filter. Let $[\Phi]$ be the equivalence class of all Cauchy filters equivalent to $\Phi$, and let $S^{*}=\{[\Phi]: \Phi$ a Cauchy filter $\}$. For $p \in S$, let $\dot{p}$ denote the fixed ultrafilter generated by $\{p\}$. The canonical map $j: S \rightarrow S^{*}$ is given by $j(p)=[\dot{p}]$. Before defining a probabilistic quasi-metric $\mathscr{F}^{*}$ on $S^{*}$, we need the following lemma.

LEMMA 5.2. Let $(S, F, \tau)$ be a PQM space. If $\Phi, \Psi$ are Cauchy filters, then $\mathscr{F}(\Phi \times \Psi)$ is a Cauchy filter relative to $\left(\Delta^{+}, d_{L}\right)$.

ProOF. It must be shown that $d_{L}[\mathscr{F}(\Phi \times \Psi) \times \mathscr{F}(\Phi \times \Psi)] \rightarrow 0$ in $R$. Let $\delta>0$ be given. By [4, Lemma 12.2.1], there exists $\eta>0$ such that, if $F, G, H \in \Delta^{+}$, with $F \geq \tau(H, G), G \geq \tau(H, F)$, and $d_{L}\left(H, \varepsilon_{0}\right)<\eta$, then $d_{L}(F, G)<\delta$.

Since $\mathscr{F}(\Phi \times \Phi) \rightarrow \varepsilon_{0}, \mathscr{F}(\Psi \times \Psi) \rightarrow \varepsilon_{0}$, and $\tau$ is continuous at $\left(\varepsilon_{0}, \varepsilon_{0}\right)$, we can choose $A \in \Phi, B \in \Psi$, such that $\tau[\hat{\mathscr{F}}(A \times A) \times \hat{\mathscr{F}}(B \times B)] \subseteq N_{\varepsilon_{0}}(\eta)$. Let $p, p^{\prime} \in A$ and $q, q^{\prime} \in B$. Then

$$
\begin{aligned}
F_{p^{\prime} q^{\prime}} & \geq \tau\left(F_{p^{\prime} q}, F_{q q^{\prime}}\right) \geq \tau\left[\tau\left(F_{p^{\prime} p}, F_{p q}\right), F_{q q^{\prime}}\right]=\tau\left[\tau\left(F_{p^{\prime} p}, F_{q q^{\prime}}\right), F_{p q}\right] \\
& \geq \tau\left[\tau\left(F_{p^{\prime} p} \wedge F_{p p^{\prime}}, F_{q^{\prime} q} \wedge F_{q q^{\prime}}\right), F_{q p}\right] .
\end{aligned}
$$

Similarly, $F_{p q} \geq \tau\left[\tau\left(F_{p p^{\prime}} \wedge F_{p^{\prime} p}, F_{q q^{\prime}} \wedge F_{q^{\prime} q}\right), F_{p^{\prime} q^{\prime}}\right]$. Since $\tau[\hat{F}(A \times A) \times$ $\hat{\mathscr{F}}(B \times B)] \subseteq N_{\varepsilon_{0}}(\eta)$, it follows that $\tau\left(F_{p p^{\prime}} \wedge F_{p^{\prime} p}, F_{q q^{\prime}} \wedge F_{q^{\prime} q}\right) \in N_{\varepsilon_{0}}(\eta)$. Let $F=F_{p^{\prime} q^{\prime}}, G=F_{p q}, H=\tau\left(F_{p p^{\prime}} \wedge F_{p^{\prime} p}, F_{q q^{\prime}} \wedge F_{q^{\prime} q}\right)$; then by [4, Lemma 12.2.1] $d_{L}\left(F_{p^{\prime} q^{\prime}}, F_{p q}\right)<\delta$. This implies that $\left.d_{L}(F(A \times B)) \times \mathscr{F}(A \times B)\right) \subseteq[0, \delta]$, and thus $\mathscr{F}(\Phi \times \Psi)$ is a Cauchy filter on $\left(\Delta^{+}, d_{L}\right)$.

Returning to the construction of a PQM completion of a PQM space $(S, \mathscr{F}, \tau)$, we define $\mathscr{F}^{*}: S^{*} \times S^{*} \rightarrow \Delta^{+}$as follows: $\mathscr{F}^{*}([\Phi],[\Psi])=\lim \mathscr{F}(\Phi \times \Psi)$ in 
$\left(\Delta^{+}, d_{L}\right)$. The preceding lemma implies that this limit exists for any Cauchy filters in the given equivalence classes; furthermore, this limit is well defined because each equivalence class contains a smallest (coarsest) filter. Note that $\mathscr{F}^{*}([\dot{p}],[\dot{q}])=\mathscr{F}(p, q)$. It will be convenient to write $F_{[\Phi][\Psi]}^{*}$ in place of $\mathscr{F}^{*}([\Phi],[\Psi])$.

Proposition 5.3. If $(S, \mathscr{F}, \tau)$ is a PQM space, then $\left(S^{*}, \mathscr{F}^{*}, \tau\right)$ is also a PQM space.

PROOF. All parts are straightforward to verify except the triangle inequality. Let $[\Phi],[\Psi],[\Gamma]$ belong to $S^{*}$; we shall show that $F_{[\Phi][\Psi]}^{*} \geq \tau\left(F_{[\Phi][\Gamma]}^{*}, F_{[\Gamma][\Psi]}^{*}\right)$. It is sufficient to show that $F_{[\Phi][\Psi]}^{*}(x) \geq \tau\left(F_{[\Phi][\Gamma]}^{*}, F_{[\Gamma][\Psi]}^{*}\right)(x)$ when $x$ is a point of continuity of $F_{[\Phi][\Psi]}^{*}$ and $\tau\left(F_{[\Phi][\Gamma]}^{*}, F_{[\Gamma][\Psi]}^{*}\right)$. Since we are dealing with Cauchy filters for a metrizable uniform space $(S, \hat{\mathscr{U}})$, we can formulate the argument in terms of sequences. Let $\left(p_{n}\right)$ be a sequence in $S$, such that $\left\langle p_{n}\right\rangle \in[\Phi]$, where $\left\langle p_{n}\right\rangle$ is the filter generated by $\left(p_{n}\right)$; similarly, let $\left\langle q_{n}\right\rangle \in[\Psi]$, and $\left\langle r_{n}\right\rangle \in[\Gamma]$. Then $F_{p_{n} q_{n}} \geq \tau\left(F_{p_{n} r_{n}}, F_{r_{n} q_{n}}\right)$, and so $F_{[\Phi][\Psi]}^{*}(x) \geq \tau\left(F_{[\Phi][\Gamma]}^{*}, F_{[\Gamma][\Psi]}^{*}\right)(x)$. Thus, $\left(S^{*}, \mathscr{F}^{*}, \tau\right)$ is a $\mathrm{PQM}$ space.

Since, as we have noted, $\mathscr{F}$ and $\hat{\mathscr{F}}=\mathscr{F} \wedge \mathscr{F}^{\prime}$ have the same Cauchy filters, the completion sets for $(S, \mathscr{F}, \tau)$ and $(S, \hat{F}, \tau)$ are the same set $S^{*}$ described above. Note that Lemma 5.2 is valid for $(S, \hat{\mathscr{F}}, \tau)$ as well as for $(S, \mathscr{F}, \tau)$; indeed, for $(S, \hat{F}, \tau)$ the conclusion follows by [4, Theorem 12.2.2]. If the construction which was applied to $\mathscr{F}$ in order to obtain $\mathscr{F}^{*}$ is applied instead to $\hat{\mathscr{F}}$, we obtain a PM space $\left(S^{*},(\hat{\mathscr{F}})^{*}, \tau\right)$, which is indeed the Sherwood completion of $(S, \hat{F}, \tau)$.

Proposition 5.4. For any PQM space $(S, \mathscr{F}, \tau),(\hat{\mathscr{F}})^{*}=\left(\hat{\mathscr{F}}^{*}\right)$.

PROOF. We must show that, for arbitrary $[\Phi],[\Psi] \in S^{*},(\hat{F})_{[\Phi][\Psi]}^{*}=F_{[\Phi][\Psi]}^{*} \wedge$ $F_{[\Psi][\Phi]}^{*}$. The verification is straightforward using $\left\langle p_{n}\right\rangle \in[\Phi]$ and $\left\langle q_{n}\right\rangle \in[\Psi]$.

Corollary 5.5. If $(S, \mathscr{F}, \tau)$ is a PQM space, then $\left(S^{*}, \mathscr{F}^{*}, \tau\right)$ is a PQM completion relative to the embedding map $j: S \rightarrow S^{*}$.

PROOF. The map $j$ is clearly an isometry (see paragraph preceding Proposition 5.3) of $(S, \mathscr{F}, \tau)$ into the PQM space $\left(S^{*}, \mathscr{F}^{*}, \tau\right)$. Since $(\hat{\mathscr{F}})^{*}$ is complete, it follows by Proposition 5.4 that $\left(\hat{\mathscr{F}}^{*}\right)$ is complete. Since $\left(\hat{\mathscr{F}}^{*}\right)$ and $\mathscr{F}^{*}$ have the same Cauchy filters and the strong topology for $\mathscr{F}^{*}$ is coarser than that for $\left(\hat{\mathscr{F}}^{*}\right)$, it follows that $\left(S^{*}, \mathscr{F}^{*}, \tau\right)$ is also complete. 
THEOREM 5.6. Every OPM space $(S, \mathscr{G}, \tau, \leq)$ has OPM completion.

Proof. Let $\left(S, \mathscr{F}, \tau^{\prime}\right)$ be a PQM space compatible with $(S, \mathscr{G}, \tau, \leq)$. If $\mathscr{U}$ is the strong quasi-uniformity for $\mathscr{F}$, then $\hat{\mathscr{U}}=\mathscr{U} \vee \mathscr{U}^{-1}$ is the strong uniformity for $\mathscr{G}$. Let $\left(S^{*}, \mathscr{G}^{*}, \tau\right)$ be the PM completion of $(S, \mathscr{G}, \tau)$; by our previous remarks, we can assume that the same set $S^{*}$ is the underlying set for the PQM completion $\left(S^{*}, \mathscr{F}^{*}, \tau^{\prime}\right)$ of $\left(S, \mathscr{F}, \tau^{\prime}\right)$. Furthermore, the strong uniformity for $\left(S^{*}, \mathscr{G}^{*}, \tau\right)$ is $(\hat{\mathscr{U}})^{*}$, where $\left(S^{*},(\hat{\mathscr{U}})^{*}\right)$ is the uniform completion of $(S, \hat{\mathscr{U}})$.

Let $\mathscr{U}^{*}$ be the strong quasi-uniformity for $\left(S^{*}, \mathscr{F}^{*}, \tau^{\prime}\right)$. By Proposition 5.4, $(\hat{\mathscr{U}})^{*}=\mathscr{U}^{*} \vee\left(\mathscr{U}^{*}\right)^{-1}$. Thus $\mathscr{G}^{*}$ is compatible with $\mathscr{F}^{*}$. Let $\leq^{*}$ be the order on $S^{*}$ determined by $\mathscr{F}^{*}$. Since $\mathscr{F}(p, q)=\varepsilon_{0}$ if and only if $\mathscr{F}^{*}([\dot{p}],[\dot{q}])=\varepsilon_{0}$, the embedding map $j:(S, \mathscr{G}, \tau, \leq) \rightarrow\left(S^{*}, \mathscr{G}^{*}, \tau, \leq^{*}\right)$ is an order-preserving isometry. Thus $\left(S^{*}, \mathscr{G}^{*}, \tau, \leq^{*}\right)$ is an OPM completion of $(S, \mathscr{G}, \tau, \leq)$.

A function $f:(S, \mathscr{U}) \rightarrow(T, \mathscr{V})$ between two quasi-uniform spaces is called uniformly continuous when $f^{-1}(V) \in \mathscr{U}$ for each $V \in \mathscr{V}$. The following is an extension result to the completion space for an increasing, uniformly continuous function.

PROPOSITION 5.7. Let $f$ be an increasing function from the OPM space $(S, \mathscr{G}, \tau, \leq)$ into the complete OPM space $\left(T, \mathscr{K}, \tau_{1}, \leq_{1}\right)$. Suppose that $\left(S, \mathscr{F}, \tau^{\prime}\right)$ and $\left(T, \mathscr{H}, \tau_{1}^{\prime}\right)$ are compatible $P Q M$ spaces with corresponding strong quasiuniformities $\mathscr{U}$ and $\mathscr{V}$, respectively. If $f:(S, \mathscr{U}) \rightarrow(T, \mathscr{V})$ is uniformly continuous, then the uniformly continuous extension to the completion $\left(S^{*}, \mathscr{G}^{*}, \tau^{*}, \leq^{*}\right)$ is also increasing.

Proof. Suppose that $[\Phi] \leq^{*}[\Psi]$; then $F_{[\Phi][\Psi]}^{*}=\lim \mathscr{F}(\Phi \times \Psi)=\varepsilon_{0}$ and this implies that $\mathscr{U} \subseteq \Phi \times \Psi$. Since $f$ is uniformly continuous, $\mathscr{V} \subseteq f \Phi \times f \Psi$ and thus $\mathscr{H}(\lim f \Phi, \lim f \Psi)=\lim \mathscr{H}(f \Phi \times f \Psi)=\varepsilon_{0}$. It follows that the uniformly continuous extension of $f$ to $\left(S^{*}, \mathscr{G}^{*}, \tau^{*}, \leq^{*}\right)$ is increasing.

\section{Acknowledgment}

The authors are indebted to the referee for suggesting numerous improvements in the original manuscript. In particular, the generalization of Proposition 3.2 from $(S, \hat{\mathscr{F}}, \tau, \leq)$ to $\left(S, \mathscr{F}^{*}, \tau, \leq\right)$ is due to the referee and, moreover, Corollary 4.6 is entirely his observation. 


\section{References}

[1] P. S. Marcus, 'Probabilistic metric spaces constructed from stationary Markov chains', Aequationes Math. 15 (1977), 169-171.

[2] L. Nachbin, Topology and order (Van Nostrand Math. Studies, No. 4, Princeton, N.J, 1965).

[3] H. Sherwood, 'On the completion of probabilistic metric spaces', Z. Wahrsch. Verw. Gebiete 6 (1966), 62-64.

[4] B. Schweizer and A. Sklar, Probabilistic metric spaces (North-Holland, New York, 1983).

[5] _ - 'Triangle inequalities in a class of statistical metric spaces', J. London Math. Soc. 38 (1963), 401-406.

Department of Pure and

Applied Mathematics

Washington State University

Pullman, Washington 99164

U.S.A.
Departments of Mathematics and Statistics

University of Central Florida

Orlando, Florida 32816

U.S.A. 